\title{
Numerical Optimization for High Efficiency, Low Noise Airfoils
}

\author{
N. Bizzarrini \\ F. Grasso \\ D.P. Coiro
}

29th AIAA Applied Aerodynamics Conference, 27-30 June 2011, Honolulu, HI, USA, AIAA 2011-3187

July 2011

ECN-M--11-068 

$29^{\text {th }}$ AIAA Applied Aerodynamics Conference

Propeller, Rotorcraft, Wind Turbine Aerodynamics

\title{
Numerical Optimization for High Efficiency, Low Noise Airfoils
}

\author{
N. Bizzarrini ${ }^{1}$ \\ University of Napoli "Federico II", 80125, Napoli, Italy \\ F. Grasso ${ }^{2}$ \\ Energy Research Centre of the Netherlands (ECN), 1755 LE, Petten, The Netherlands \\ and \\ D. P. Coiro ${ }^{3}$ \\ University of Napoli "Federico II", 80125, Napoli, Italy
}

\begin{abstract}
One key element in the aerodynamic design of wind turbines is the use of specially tailored airfoils to increase the ratio of energy capture to the loading and thereby to reduce cost of energy. At the same time, noise reduction is becoming more and more important. This work is focused on the design of a silent wind turbine airfoil by using numerical optimization. Firstly, the optimization approach is presented; a genetic algorithm is used, coupled with RFOIL, NAFNOISE solvers and a composite Bezier geometrical parameterization. A particularly sensitive point is the choice and implementation of constraints; in order to formalize in the most complete and effective way the design requirements, the effects of activating specific constraints are discussed. Finally, a numerical example regarding the design of a high efficiency, silent airfoil for the outer part of a blade is illustrated and the results are compared with existing wind turbine airfoils.
\end{abstract}

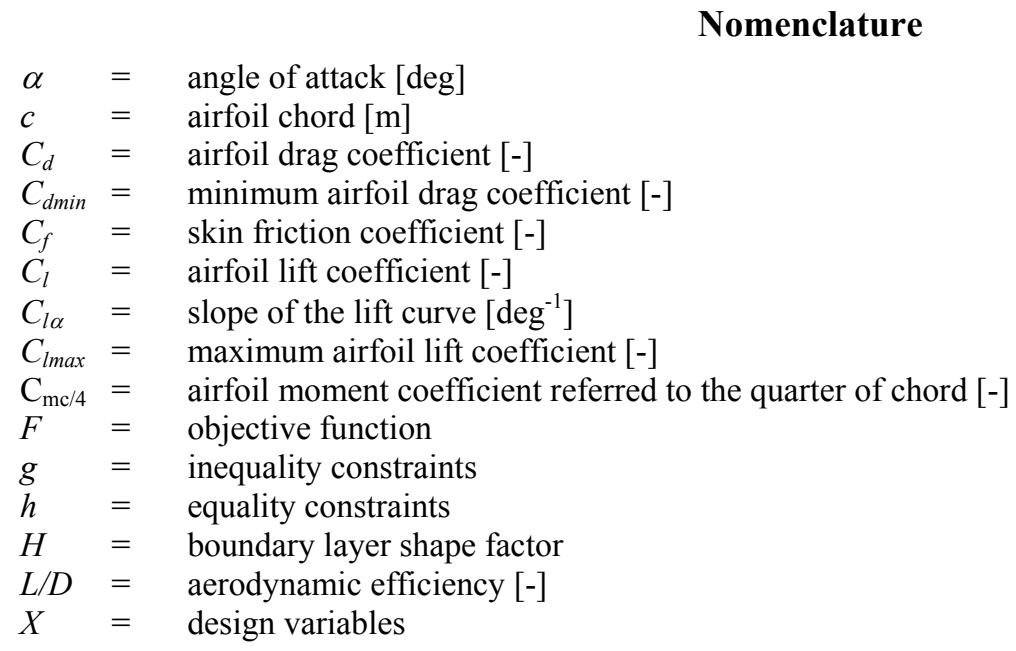

\footnotetext{
${ }^{1}$ MSc graduating student, Department of Aerospace Engineering, via Claudio 21; nadia.bizzarrini@gmail.com

${ }^{2}$ Postdoctoral Aerodynamicist, Wind Energy Unit, Rotor and Wind Farms Aerodynamics Group, Westerduinweg 3; grasso@ecn.nl, Associate Fellow AIAA.

${ }^{3}$ Associate Professor, Department of Aerospace Engineering, via Claudio 21; coiro@unina.it, AIAA member.
} 
$X^{L} \quad=\quad$ lower bounds for the design variables

$X^{U}=$ upper bounds for the design variables

\section{Introduction}

DEIGN of airfoils specifically suited for wind turbine blade applications is important in the continuing development of wind turbines. New airfoils families [1-4] for wind turbines are developed because of the intrinsic requirements in terms of design point, off-design capabilities and structural properties.

The target design characteristics for the airfoils have been updated during the years and tailored to the specific type of power control and the need for off-design operation. The desirable airfoil characteristics can be divided into structural and aerodynamic properties and the wind turbine blade can be divided into the root, mid and tip parts where the root part is mainly determined from structural considerations. In contrast, the tip part is determined from aerodynamic considerations. In addition to this, due to the development of wind energy in densely populated areas, the reduction of the noise generated by wind turbines is becoming an important requirement. During the last years, several projects were focused on this topic. In particular, the SIlent ROtors by aCoustic Optimization (SIROCCO) project [5] pointed the attention on the fact that designing silent airfoils can be the most effective way to reduce the noise of the rotating blades.

This work is focused on the design of a silent, wind turbine dedicated airfoil for the tip region of the blade, by using numerical optimization.

\section{Numerical Optimization Approach}

Especially when multiple requirements, concerning different disciplines are involved in the design process, it can be convenient to use multidisciplinary design optimization (MDO) approach. In the most general sense, numerical optimization $[6,7]$ solves the nonlinear, constrained problem to find the set of design variables, $X_{i}, \mathrm{i}=1, \mathrm{~N}$, contained in vector $\boldsymbol{X}$, that will:

\section{Minimize $F(X)$}

subject to:

$$
\begin{aligned}
& g_{j}(X) \leq 0 \quad j=1, M(2) \\
& h_{k}(X)=0 \quad k=1, L \\
& X_{i}^{L} \leq X_{i} \leq X_{i}^{U} \quad i=1, N
\end{aligned}
$$


Equation 1 defines the objective function which depends on the values of the design variables, $\boldsymbol{X}$. Equations 2 and 3 are inequality and equality constraints respectively (equality constraints can be written as inequality constraints and included in equation 2), and equation 4 defines the region of search for the minimum. The bounds defined for each degree of freedom by equation 4 are referred to side constraints.

\section{A. Geometry Description}

One of the most important ingredients in numerical optimization is the choice of design variables and the parameterization of our system in using these variables. In order to reduce the number of necessary parameters to take into account to describe the airfoil's shape, but without loss of information about the geometrical characteristics of the airfoil, several mathematical formulations were proposed in literature [8]. In the present work, a composite third order Bezier is used. Basically, the airfoil is divided in four parts and for each part, a third order Bezier curve is used to describe the geometry. The advantage of this choice is the possibility to conjugate the properties of Bezier functions in terms of regularity of the curve and easy usage, with a piecewise structure that allows also local modifications to the geometry. The complete description can be found in [9]. In this work, eight degrees of freedom are actively used, which correspond to the ordinates of the control points $2,3,5,6,8,9,11,12$. The control points 4 and 10 are automatically controlled by the algorithm in order to ensure curvature continuity between different Bezier curves.

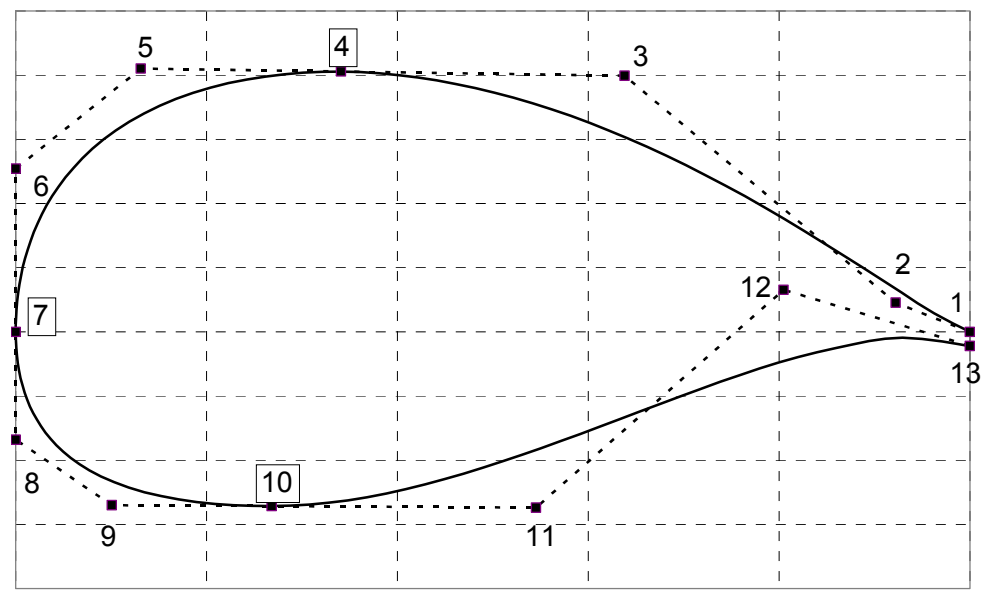

Fig. 1 Geometry parameterization example. 


\section{B. Optimization Algorithm}

The choice of optimization algorithm is very important because the final results are usually dependent on the specific algorithm in terms of accuracy and local minima sensitivity. In this work, Genetic Algorithm (GA) developed by Caroll $[10,11]$ is used for the optimization process. The main reason of this choice is the capability of GA to explore wide, non-linear and discontinuous domains and be less sensitive to the initial configuration, especially when compared to gradient-based algorithms (GBA). In addition to this, GBA are more accurate, but also more sensitive to local optima for complex domains. Due to the multidisciplinary context, GA were preferred at this stage, even if with less accuracy than GBA. In the implemented GA, several evolutionary mechanisms are included and here, briefly summarized. The complete description of the algorithm and the parameters can be found in [10].

\section{Selection}

Tournament Selection scheme is used. In practice, random pairs are selected from the population and the most fit of each pair is allowed to mate. Each pair of mates create a number of offspring that have some mix of the two parents chromosomes according the method of crossover. The process continues until a new generation of $n$ individuals is created.

\section{Crossover}

In this work uniform crossover is chosen where each bit has a probability for a crossover with the second parent, so it is possible to obtain every combination of the two parents.

\section{Mutation}

In order to prevent the solution from local optima, a new individual point in the solution space is created by altering one of the bits of an individual. In the present research, both jump mutation and creep mutation are used. In jump mutation one or more child's chromosomes have a probability to be subjected to a mutation not depending on either parent. In particular a child's parameter is randomly selected and then its value is changed by a random amount chosen within a prescribed range. In creep mutation one or more child's parameters have a probability to be incremented up or down from the relative parent value.

\section{Elitism}

To guarantee convergence, the chromosome set of the best parent is reproduced in the succeeding generation.

\section{MicroGA}

The MicroGA is a method to improve the performance of genetic algorithms used in this work and derived by the studies of Goldberg and Krishnakumar [12] to explore the use of small population sizes in genetic. Small populations can lead to too rapid convergence, so the re-generation of random population members helps to ensure diversity during the search process and so to avoid local minima. 


\section{Objective Function Evaluation}

Since the optimization process requires many evaluations of the objective function and the constraints before an optimum design is obtained, the computational costs cannot be neglected, as well as the accuracy of the results.

\section{RFOIL}

RFOIL [13] is a modified version of XFOIL [14] featuring an improved prediction around the maximum lift coefficient and capabilities of predicting the effect of rotation on airfoil characteristics. Regarding the maximum lift in particular, numerical stability improvements were obtained by using the Schlichting velocity profiles for the turbulent boundary layer, instead of Swafford's. Furthermore, the shear lag coefficient in Green's lag entrainment equation of the turbulent boundary layer model was adjusted and deviation from the equilibrium flow has been coupled to the shape factor of the boundary layer. The following figures illustrate a comparison with experimental data [15] for the NACA-63, 418 airfoil. The Reynolds number is 6 million and the transition is free.

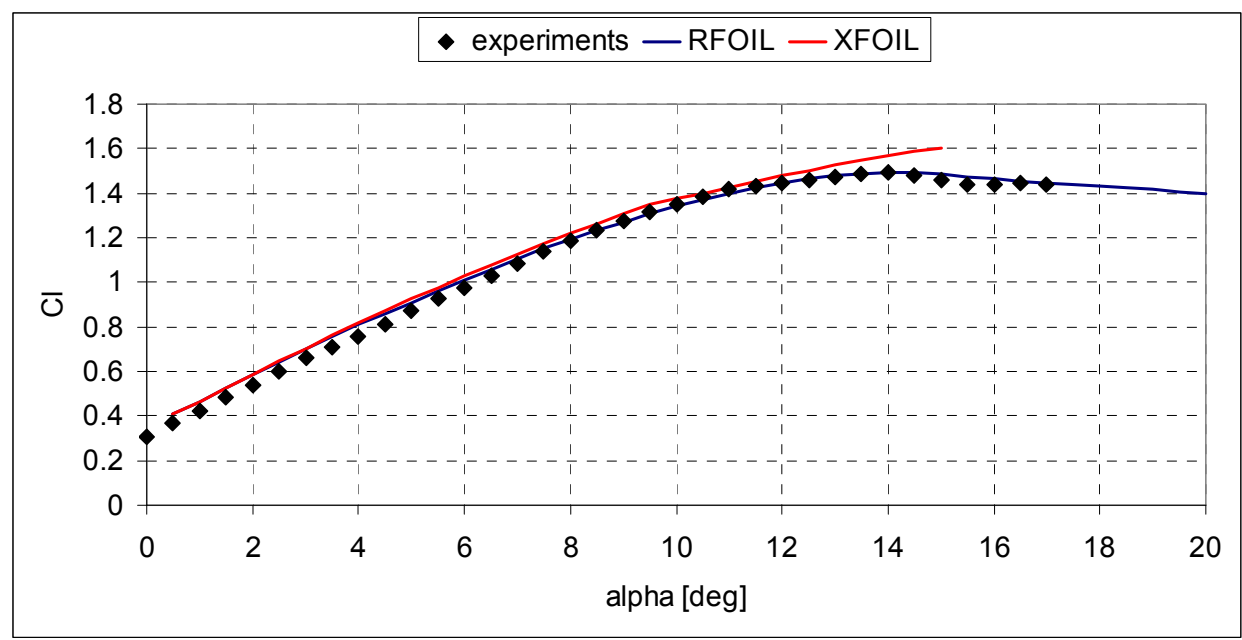

Fig. 2 Lift curve for the NACA- $63_{3} 418$ airfoil; comparison between XFOIL and RFOIL with experiments [15]. Reynolds number 6 million, free transition. 


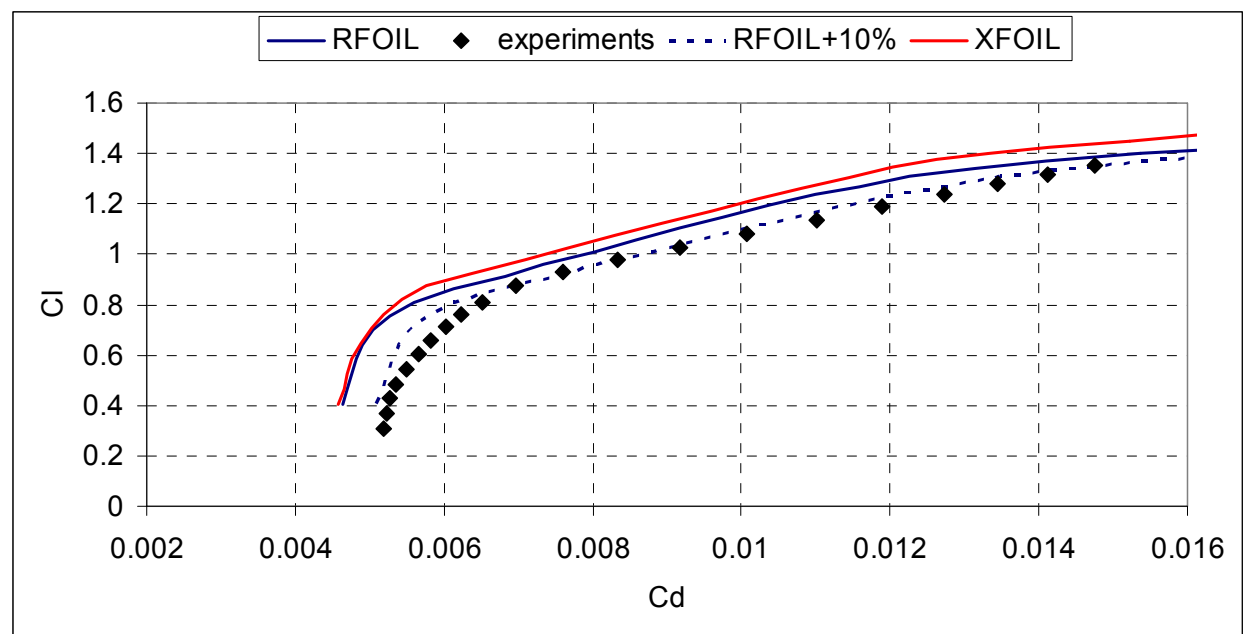

Fig. 3 Drag curve for the NACA-63 418 airfoil; comparison between XFOIL and RFOIL with experiments [15]. Reynolds number 6 million, free transition.

It should be noted that the RFOIL prediction for the stall region is well described and very close to the experimental data; in XFOIL results, only the deviation from the linear zone is described but not the stall. For the drag curve, XFOIL and RFOIL are very close to each other for small values of $C_{l}$, but for high $C_{l}$, XFOIL is under predicting. In ref [13], a additional drag of $10 \%$ is suggested to correct the RFOIL data. In order to have more realistic predictions, this $10 \%$ drag penalty is added during the optimization process and for all the numerical analyses.

\section{NAFNOISE}

NAFNoise (NREL AirFoil Noise) is a program developed at the National Renewable Energy Laboratory (NREL) that predicts the noise of any airfoil shape for five different types of noise sources by using several mathematical models [16- 20]: turbulent boundary layer trailing-edge noise, separated flow noise, laminar boundary layer vortex-shedding noise, trailing-edge bluntness noise, and turbulent inflow noise. In the present work only the turbulent boundary layer trailing edge noise is considered because is widely predominant on the other ones and the model developed by Brooks, Pope and Marcolini (BPM) [20] is used. This model is based on detailed measurements of noise from NACA 0012 airfoils and it handles every kind of noise sources except turbulent inflow. The boundary layer properties can be calculated by using empirical relations, or retrieve these data from XFOIL calculations. Here, this second option has been preferred because the actual airfoil shape can be taken into account. 


\section{Design of tip region airfoil}

The design of a new airfoil for the tip region of the blade is presented in this section. A complete discussion about the design requirements for this class of airfoils is presented in [21]. In order to exhaustively describe the problem, both geometrical and aerodynamic requirements need to be matched; several design and off-design conditions should be taken into account, as well as the characteristics of the other airfoils mounted on the same blade. In this work, only part of these requirements has been included because the main interest was focused on the role and effects of acoustics on aerodynamic performance.

The new airfoil is designed to maximize the aerodynamic efficiency and, at the same time, to reduce the noise at 6 degrees of angle of attack, at Reynolds number of 1 million and a Mach number of 0.189 .

The unit of measurement used for the noise level is the so called "A-weighted dB", which is based on a weighting curve modelled on the ear's response to low and high levels of sound respectively.

Aerodynamic efficiency and noise level are combined together in weighted sum. The optimization process has been repeated for several values of the weight factor in order to investigate the sensitivity of the solution to the relative importance of the aerodynamics and acoustics.

With regard to GA parameters the following settings are used: tournament selection, uniform crossover, jump and creep mutations, elitism and microGA strategy; number of individual of each generation equal to 5 , number of generations equal to 200 , jump mutation probability equal to 0.02 , creep mutation probability equal to 0.04 , number of children for each couple of parents equal to 1.

\section{A. Geometrical Constraints}

Usually, the thickness at the tip is between $15 \%$ and $18 \%$ of the chord; here a minimum value of $18 \%$ is prescribed and a chord-wise location around $30 \%$ of the chord in order to be compatible with existing airfoils.

A minimum trailing edge thickness of $0.25 \%$ of the chord is required to ensure airfoil's feasibility from manufacturing point of view.

\section{B. Aerodynamic Constraints}

In [21], in order to limit the torsion of the blade, a minimum value for $C_{m c / 4}$ of -0.08 is prescribed. This value comes from a comparative analysis on experimental data $[3,16]$ for existing airfoils realistically usable for this class of turbines. However, in the present work, this constraint has been not activated. The aim of this choice is to have a better understanding about the effect of noise reduction on the shape of the airfoil. In fact, as discussed in [22], the 
effect on the airfoil's geometry of imposing a constraint on the $C_{m c / 4}$ is concentrated mainly at the trailing edge zone in order to limit the amount of camber and decrease the moment coefficient, but the trailing edge is also the zone of main interest for trailing edge noise.

To avoid possibility of abrupt stall and converge to a solution in which a Stratford style recompression is not present (it can lead to a not gradual evolution in transition location), the design is performed by fixing transition at $0.02 \mathrm{c}$ on the suction side and $0.1 \mathrm{c}$ on the pressure side.

\section{Results}

The optimized airfoils for several values of the weight factor of aerodynamic efficiency (in the figures indicated as $l d$ ) and noise (in the figures indicated as $n s$ ) are shown in Fig. 4, together with the NACA $63_{3} 418$ airfoil. Figures 5-10 show the comparison between the different geometries in terms of lift curves, drag curves and aerodynamic efficiency curves in both forced and free transition . According to RFOIL predictions, all the designed airfoils show good characteristics in terms of efficiency and stall behaviour. Compared to the NACA $63_{3} 418$ airfoil, the generated airfoils have a quite extended linear part of the lift curve and also better aerodynamic efficiency and off-design characteristics for fixed transition. All the airfoils are more cambered than the reference one and this is consistent with the expectations because the constraint on the moment coefficient was not part of the design problem and the natural tendency to achieve high values of aerodynamic efficiency is to increase the amount of camber. Actually, one of the generated airfoils, the one for ns $=0.8$, is closer to the NACA $63_{3} 418$ in terms of moment coefficient, with similar aerodynamic efficiency in free transition but with a better performance in terms of noise and efficiency in fixed transition.

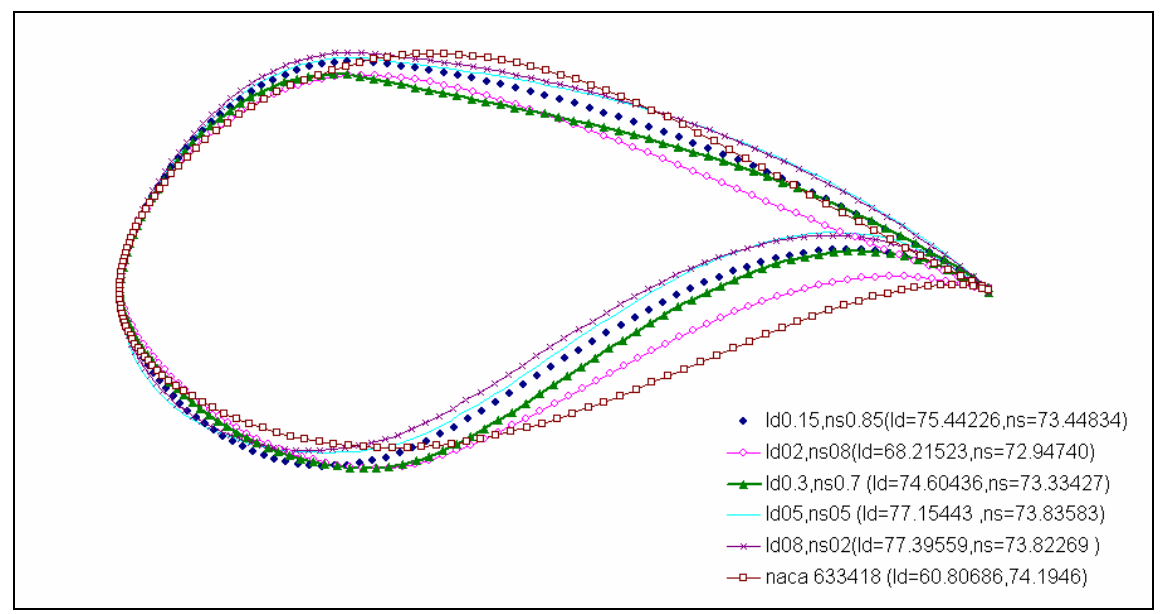

Fig. 4 Optimized airfoils for different combinations of weights of efficiency and noise. 


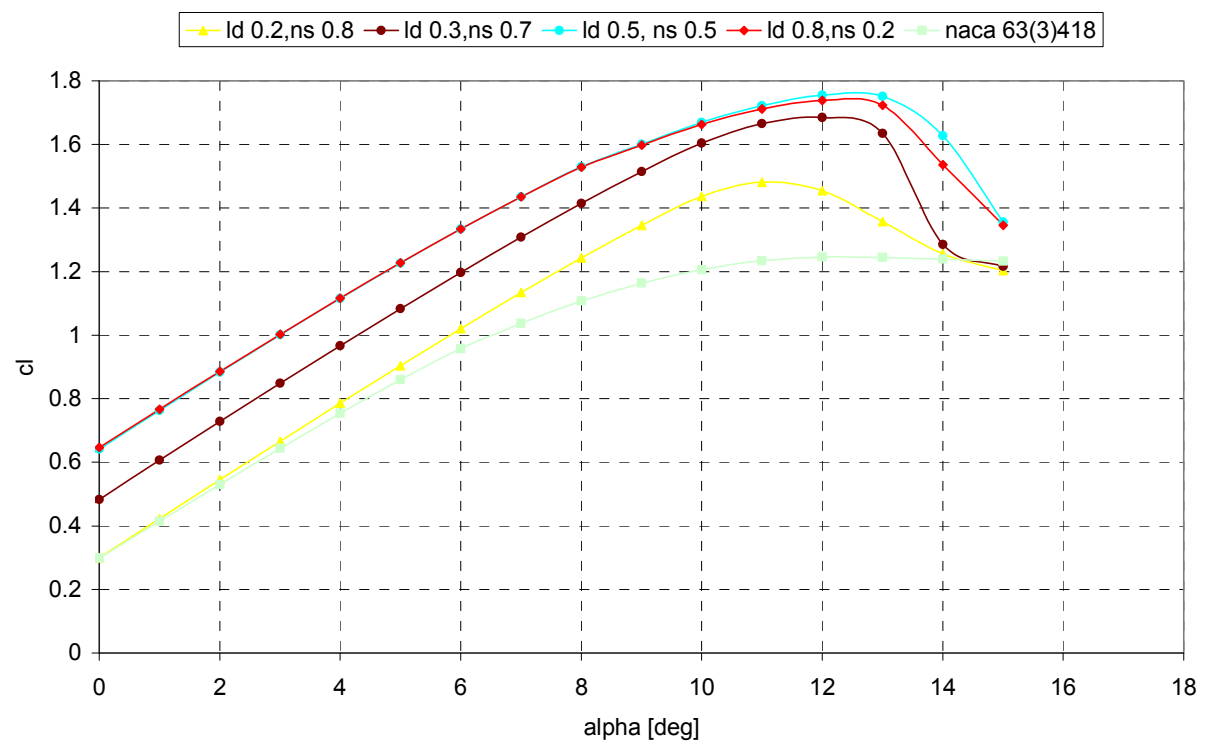

Fig. 5 Optimized airfoils for different combinations of weights of efficiency and noise. Lift curves comparison; RFoil predictions.

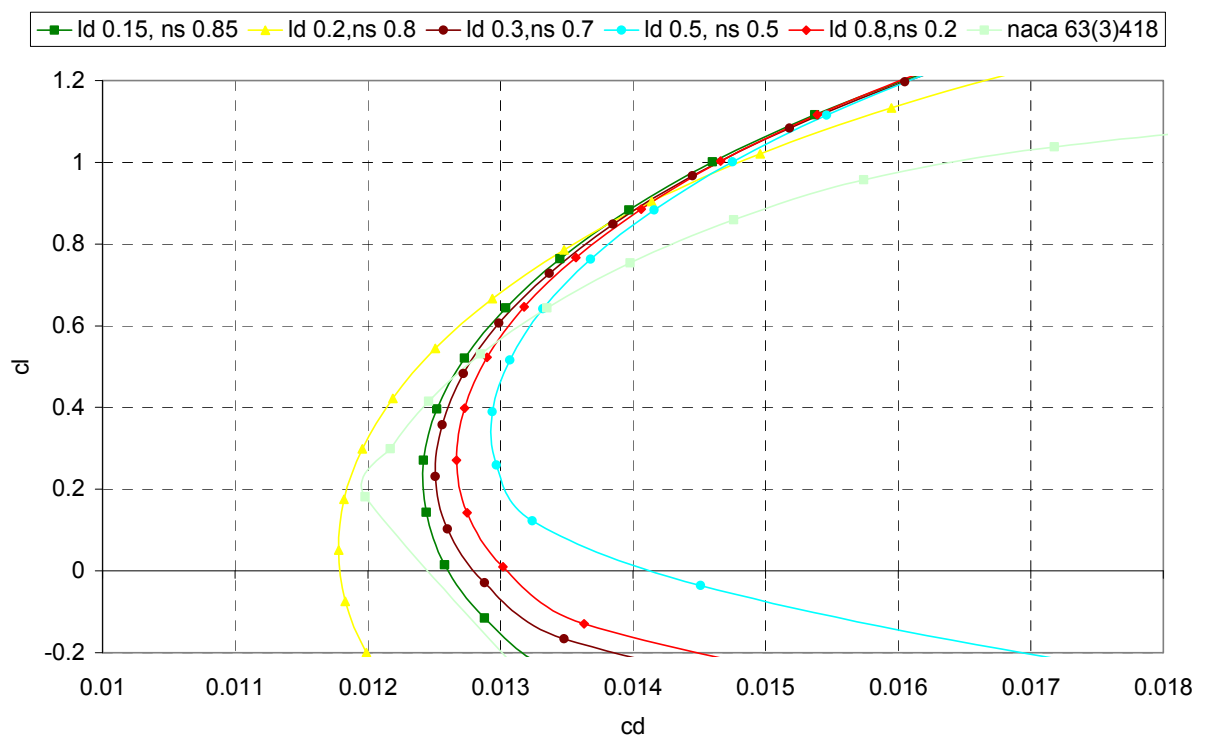

Fig. 6 Optimized airfoils for different combinations of weights of efficiency and noise. Drag curves comparison; RFoil predictions. 


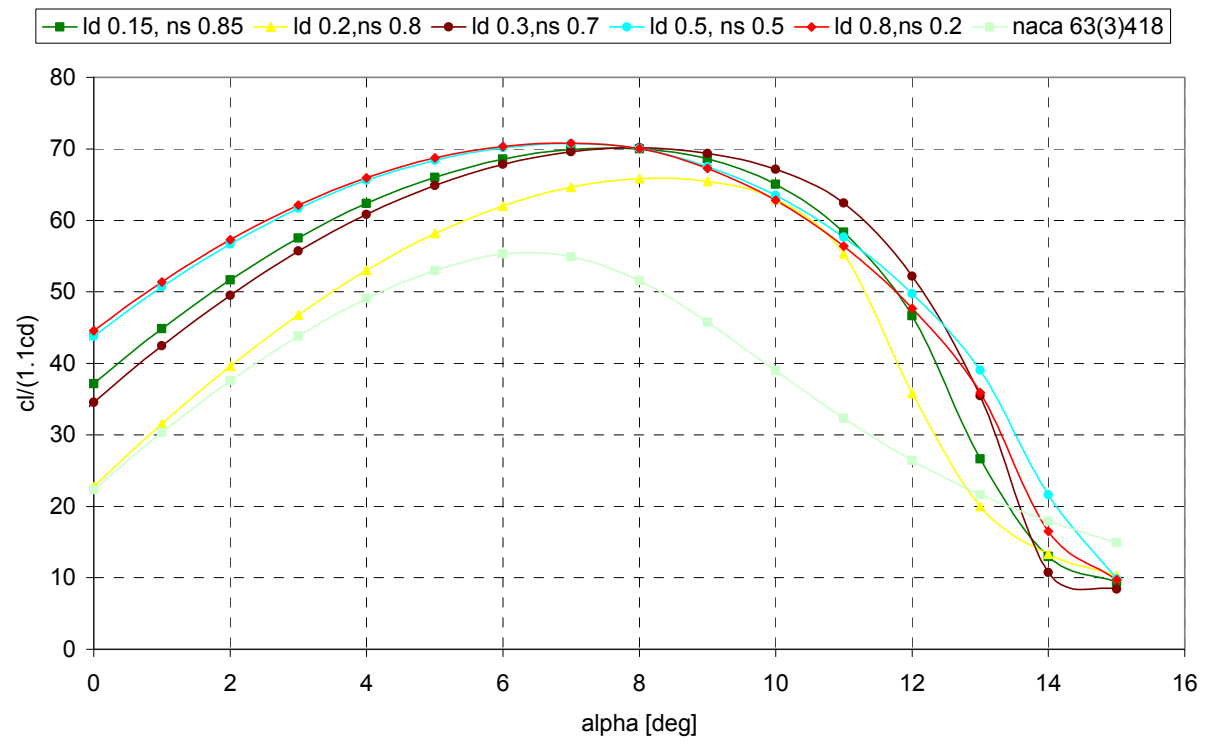

Fig. 7 Optimized airfoils for different combinations of weights of efficiency and noise. Aerodynamic efficiency curves comparison; RFoil predictions.

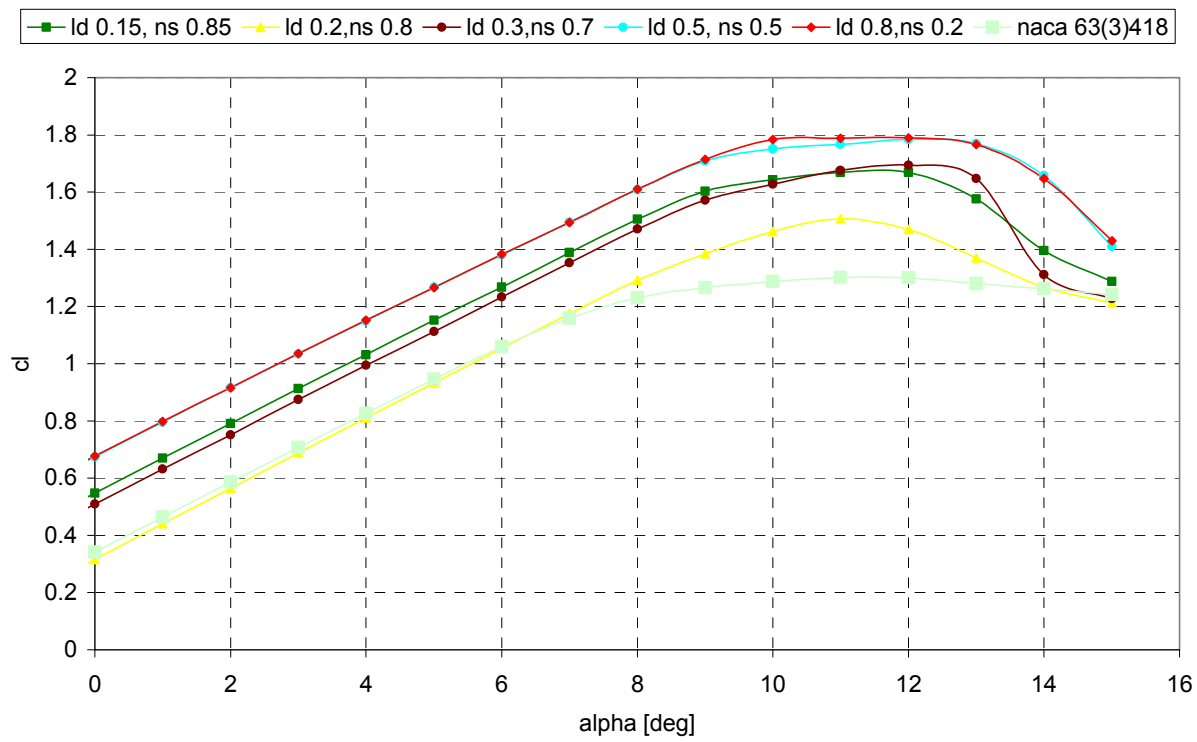

Fig. 8 Optimized airfoils for different combinations of weights of efficiency and noise. Lift curves comparison. Free transition; RFoil predictions. 


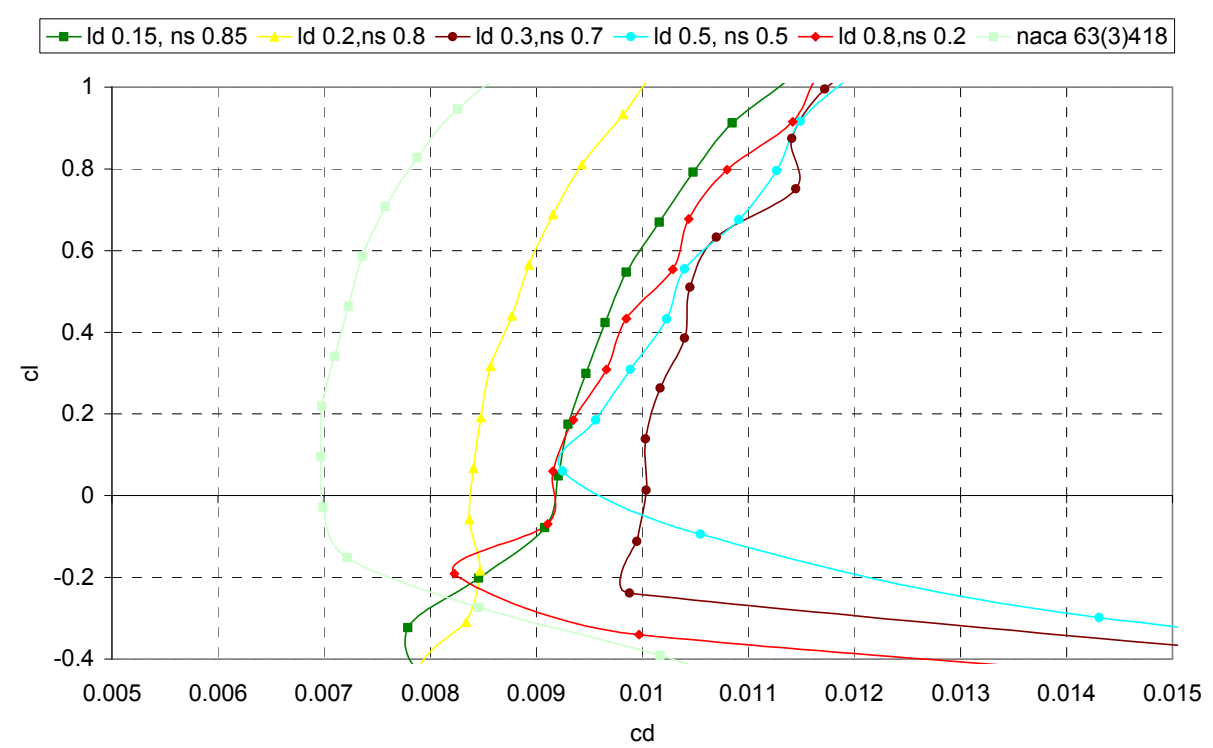

Fig. 9 Optimized airfoils for different combinations of weights of efficiency and noise. Drag polars comparison. Free transition; RFoil predictions.

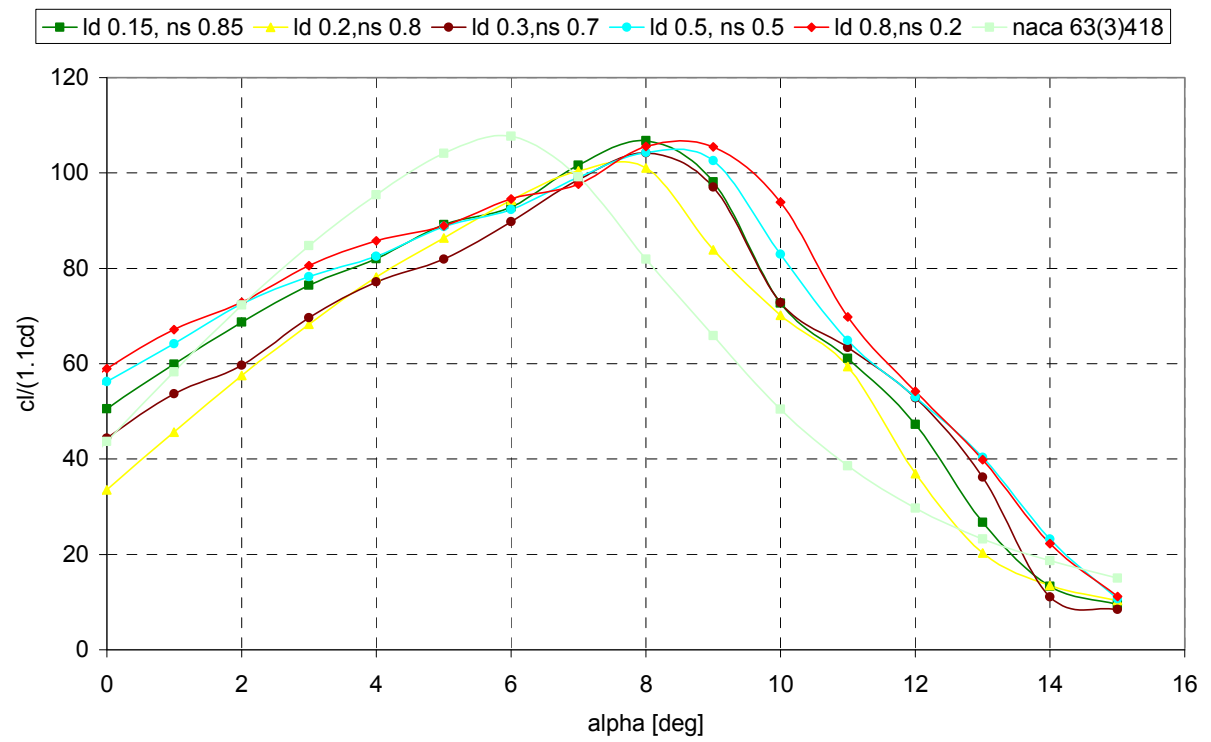

Fig. 10 Optimized airfoils for different combinations of weights of efficiency and noise. Aerodynamic efficiency curves comparison. Free transition; RFoil predictions. 


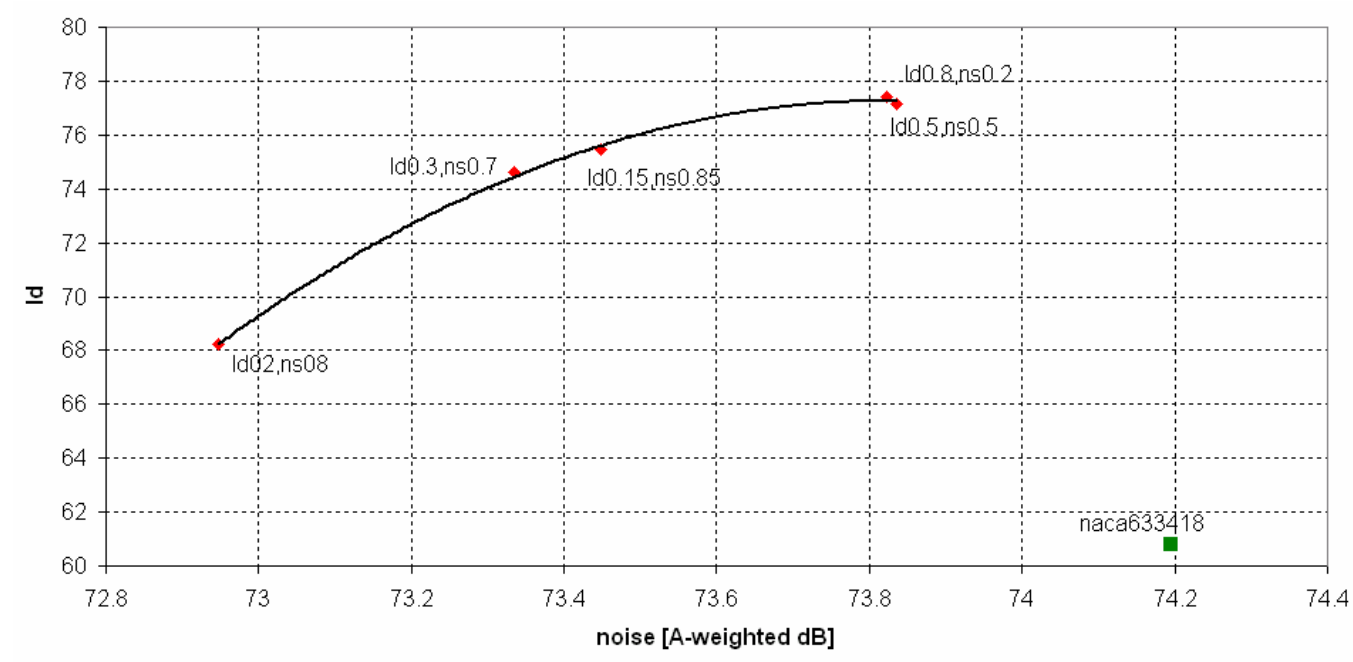

Fig. 11 Pareto curve. Aerodynamic efficiency calculated in fixed transition conditions (RFoil predictions).

Fig. 11 shows the Pareto curve for the designed airfoils. In accordance with the expectations, by changing the relative importance between aerodynamic efficiency and noise level, is possible to generate a family of airfoils in which the best candidate can be selected according to the specific assigned requirements. In the same figure, also the NACA $63_{3} 418$ airfoil is present. All the designed airfoils are dominating solutions compared to the NACA airfoil because they improve both the noise level and the efficiency. However, it should be taken into account that the moment coefficient is not part of the set of constraints; except for the generated airfoil for ns $=0.8$, all the other geometries are more cambered than the NACA $63_{3} 418$. For a real application, this will have consequences on the torsion of the blade. Also, it should be considered that the new airfoils are designed in fixed transition; the NACA 63 3418 can have better performance in clean conditions.

\section{Conclusions}

By using genetic algorithms, a family of airfoils that improves the aerodynamic efficiency and reduces the noise has been designed. According to the numerical predictions, the performances are changing gradually between the new airfoils. This allows the designer to select the most appropriate geometry for the specific application. Further developments can be done by adding more constraints in the design process.

Despite these good results, wind tunnel tests are recommended to validate predictions. Especially for the stall behaviour, the numerical predictions, and consequently the MDO process used in this work, need to be verified. 


\section{References}

[1]. Tangler, J.L., Somers, D.M., “NREL Airfoil Families for HAWT's”. Proc. WINDPOWER'95, Washington D.C., 1995; pp. $117-123$

[2]. Björk, A., "Coordinates and Calculations for the FFA-W1-xxx, FFA-W2-xxx and FFA-W3-.xxx Series of Airfoils for Horizontal Axis Wind Turbines”. FFA TN 1990-15, Stockholm, Sweden 1990

[3]. Timmer, W.,A., van Rooij, R.P.J.O.M., "Summary of the Delft University Wind Turbine Dedicated Airfoils". AIAA-20030352.

[4]. Fuglsang, P., Bak, C., "Design and Verification of the new Risø-A1 Airfoil Family for Wind Turbines". AIAA-2001-0028.

[5]. http://www.ecn.nl/units/wind/projects/sirocco/

[6]. Fletcher, R., "Practical Methods of Optimization", Wiley, 1987.

[7]. Pedregal, P., "Introduction to Optimization”, Springer, 2004, ISBN 0-387-40398-1.

[8]. Samareh, J., A., "Survey of Shape Parameterization Techniques for High-Fidelity Multidisciplinary Shape Optimization", AIAA Journal, Vol. 39, No. 5, May 2001, pp. 877-884.

[9]. Grasso, F., "Multi-Objective Numerical Optimization Applied to Aircraft Design", Ph.D. Thesis, Dip. Ingegneria Aerospaziale, Università di Napoli Federico II, Napoli, Italy, December 2008.

[10]. http://cuaerospace.com/carroll/ga.html

[11]. Yang, G., Reinstein L.E., Pai S., Xu Z., and Carroll D.L., "A new genetic algorithm technique in optimization of permanent 125-I prostate implants," Medical Physics, Vol. 25, No. 12, December 1998, pp. 2308-2315.

[12]. Krishnakumar K.,’Micro-Genetic Algorithms for Stationary and Non-Stationary Function Optimization.” SPIE: Intelligent Control and Adaptive Systems,1196, Philadelphia, PA, 1989

[13]. Van Rooij, R.P.J.O.M., "Modification of the boundary layer calculation in RFOIL for improved airfoil stall prediction", Report IW-96087R TU-Delft, the Netherlands, September 1996.

[14].Drela, M., "XFOIL 6.94 User Guide”, MIT Aero \& Astro, Dec 2001.

[15].Abbott, I., Von Doenhoff, A., “Theory of Wing Sections”, Dover Publications, Inc., Dover edition, 1958.

[16]. Brooks, T., Pope, D., and Marcolini, M., "Airfoil Self-Noise and Prediction," NASA Reference Publication 1218, National Aeronautics and Space Administration, 1989.

[17]. Amiet, R., "Acoustic Radiation from an Airfoil in a Turbulent Stream," J. Sound Vibration, Vol. 41, No. 4, pp. 407-420, 1975.

[18].Moriarty, P. and Migliore, P., Semi-Empirical Aeroacoustic Noise Prediction Code for Wind Turbines, NREL/TP-50034478, National Renewable Energy Laboratory, Golden, CO, 2003. 
[19]. Moriarty, P. , Guidati, G., Migliore, P., " Recent Improvement of a Semi-Empirical Aeroacoustic Prediction Code for Wind Turbines," Proc., 10th AIAA/CEAS Aeroacoustics Conference, Manchester, UK, AIAA 2004-3041, 2004.

[20]. Moriarty, P. , Guidati, G., Migliore, P., " Prediction of Turbulent Inflow and Trailing-Edge Noise for Wind Turbines," Proc., 11th AIAA/CEAS Aeroacoustics Conference, Monterey, California, AIAA 2005-2881, 2005.

[21]. Grasso, F., "Usage of Numerical Optimization in Wind Turbine Airfoil Design", 28 ${ }^{\text {th }}$ AIAA Applied Aerodynamics Conference, AIAA, Chicago, IL, 28 June-1 July, 2010. Also, Journal of Aircraft, Vol.48, No.1, January-February 2011, DOI: $10.2514 / 1 . C 031089$.

[22]. Bizzarrini, N., Grasso, F., Coiro, D.P., "Genetic Algorithms in Wind Turbine Airfoil Design”, EWEA, EWEC2011, Bruxelles, Belgium, 14-17 March 2011. 